\title{
Frequencies and latencies in detecting two-flash stimuli $^{1}$
}

\author{
MITCHELL GROSSBERG ${ }^{2}$ \\ THE CITY UNIVERSITY OF NEW YORK
}

\begin{abstract}
Dark-adapted Ss responded as soon as they detected near-threshold stimuli, which usually comprised two flashes that were equal in luminance and duration. The percentage of trials on which the $S$ responded and the median latency of responses were determined as functions of the interval between flash onsets. At short intervals: the percentage was independent of the interval and attributable to the stimulus energy alone (Bloch's law); the latency increased gradually to the value for the first flash alcne and was at tributable to not only the energy, but also to its temporal distribution. At long intervals: the percentage decreased to a steady level that was either equal to or higher than the value for the first flash; the latency increased to a peak and then decreased to the value for the first flash; both response measures suggested inhibition and probability summation between the flashes.
\end{abstract}

This article concerns detection experiments in which temporal characteristics of the stimulus are manipulated while the response latency is measured in conjunction with the response frequency. The near-threshold stimuli usually comprise pairs of equal flashes, the onsets of which are separated by various intervals. How the response measures covary as functions of the interflash interval is demonstrated.

In previous threshold studies with two-flash stimuli, the measure of response was either the detection frequency (Bouman \& van den Brink, 1952; van den Brink \& Bouman, 1954) or the threshold luminance (Blackwell, 1963; Davy, 1952; Ikeda, 1965). All studies indicated that the interaction between the flashes decreased and took different forms as the interflash interval increased. The studies concurred in showing complete energy summation at short intervals. That is, the behavioral effect was not only independent of the interval, but was also predictable with reference to the total stimulus energy. This behavioral constancy for a constant energy was in accord with Bloch's law. At longer intervals, where the law no longer held, the results were less uniform among studies. The details of this unexplained diversity have already been discussed by Ikeda (1965).
In the present experiments, therefore, one might expect Bloch's law to account for the frequency of response at short intervals. The outcome at longer intervals is less certain. On the other hand, the general validity of Bloch's law for response latencies is questionable. Grossberg (1968) found that the median response latency was inversely related to the luminance of brief stimuli that were constant in energy, even though the relative response frequency was constant. Aspects of this earlier work are included in the present design to bring one- and two-flash functions together within the framework of a single experiment. Three experiments are reported. They cover the range of relative response frequencies from $100 \%$ (Experiment 1), as in determinations of simple reaction times, to $20 \%$ (Experiment 3 ), as in the frequency-of-seeing determinations by Bouman and van den Brink (1952).

\section{Apparatus}

\section{METHOD}

The same apparatus was used in Grossberg's (1968) Experiment 2. In brief, the stimulus field was circular and subtended $0.5 \mathrm{deg}$. It was centered between two dim red fixation lights that were $2 \mathrm{deg}$ to the left and right, respectively. This array was viewed binocularly in darkness from a headrest. Stimulus flashes were produced by transilluminating the field with light from an electronically gated fluorescent lamp. The stimulus time characteristics were controlled with Tektronix generators (Types 161 and 162), and the stimulus luminance was controlled with neutral-density filters.

\section{Subjects}

Two male university students were paid Ss. One of them served in Experiment 1; the other served first in Experiment 2 and later in Experiment 3.

\section{Procedure}

The S's task was the same as the one assigned by Grossberg (1968). A microswitch was to be released as soon as the stimulus was detected but to be depressed continuously if no light was seen. If the switch was released within $1 \mathrm{sec}$ after stimulus onset, or the same moment on "catch" trials (when no stimulus was presented), a response was said to have occurred; its latency was measured from stimulus onset to the nearest millisecond. If the switch was not released during the $1-\mathrm{sec}$ period, the trial was ended with a signal and no latency was recorded. Thirty seconds after the end of a trial, the $S$ received a signal that informed him that he might begin a new trial whenever he was ready. He started the 2.8-sec period that preceded the stimuius period, which contained two, one, or zero flashes, according to a random schedule. The stimulus was always referred to as "the light," and the $S$ was never informed of its composition. The $S$ had been told that there would be catch trials, but these were only identified when he responded (i.e., on less than $1 \%$ of the catch trials). The $\mathrm{S}$ was adapted to darkness for $10 \mathrm{~min}$.

The two-flash stimuli consisted of successive flashes that were equal in luminance and duration. The interval between the flash onsets was the independent variable. During any block of trials, one pair of flashes was presented with various intervals that covered a wide range of values. On some of the trials, the first flash of the pair was presented alone. On other trials, no flashes were presented. That is, a standard block contained fixed numbers of randomly ordered trials with the flash pair at each of the interflash intervals, the first flash alone, and no flashes. The details of the experiments were as follows.

In Experiment 1, the two-flash stimuli comprised flashes that were $1 \mathrm{msec}$ in duration and $-1.24 \log \mathrm{ft}-\mathrm{L}$ (base 10) in luminance. The interflash intervals can be seen in Fig. 1. In the entire experiment, each interval occurred on 32 trials, and the first flash alone occurred on 64 trials; there were 315 catch trials.

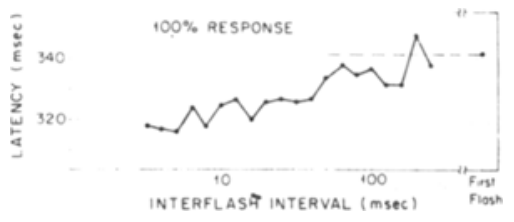

Fig. 1. The median of response latencies, as a function of the interval between two 1-msec flashes. Experiment 1. 


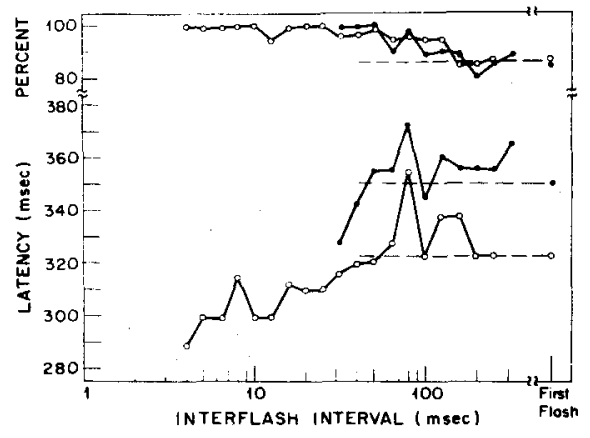

Fig. 2. The percentage of responses and the median of response latencies, as functions of the interval between two 2.5-msec flashes (unfilled circles) and two 25-msec flashes (filled circles). The two pairs of flashes were equal in energy. Experiment 2.

In Experiment 2, the two-flash stimuli comprised flashes that were either $25 \mathrm{msec}$ in duration and $-1.77 \log \mathrm{ft}-\mathrm{L}$ in luminance or $2.5 \mathrm{msec}$ in duration and $-0.74 \log \mathrm{ft}-\mathrm{L}$ in luminance; the two pairs were equal in energy. Respective interflash intervals can be seen in Fig. 2. The two pairs were presented during separate blocks, the order being counterbalanced. In the entire experiment with the 25 -msec flashes, each interval occurred on 60 trials, and the first flash alone occurred on 120 trials; with the $2.5-\mathrm{msec}$ flashes, each interval occurred on 48 trials, and the first flash alone occurred on 96 trials; there were 312 catch trials.

In Experiment 3, the two-flash stimuli comprised flashes that were $2.5 \mathrm{msec}$ in duration and $-2.58 \log \mathrm{ft}-\mathrm{L}$ in luminance. The interflash intervals can be seen in Fig. 3. Each block of trials included the standard block described previously, plus trials with stimuli that comprised single flashes; the flashes were all $-2.58 \log \mathrm{ft}-\mathrm{L}$ in luminance but ranged in duration from 4.5 to $100 \mathrm{msec}$ (Fig. 3). All stimuli were randomized together. In the entire experiment with the two-flash stimuli, each interval occurred on 54 trials, and the first flash alone occurred on 108 trials; with the one-flash stimuli, each duration occurred on 54 trials; there were 324 catch trials.

\section{RESULTS}

The S's behavior is summarized in terms of two measures: (1) the percentage of trials on which the $S$ responded and (2) the median of the response latencies. Their covariation in Experiments 1,2, and 3 is shown in Figs. 1, 2, and 3, respectively. On the abscissas are the intervals between the onsets of flashes in the two-flash stimuli. The abscissa of Fig. 3 also gives the durations of stimuli that comprised one flash. Figure 4 emphasizes the relatively

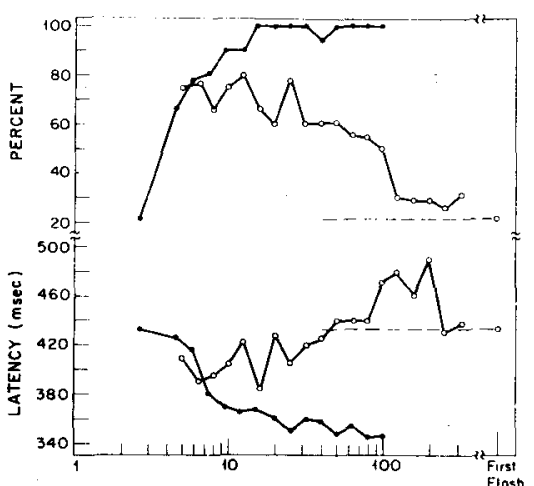

INTERFLASH INTERVAL AND FLASH DURATION ( $\mathrm{mseC}$

Fig. 3. The percentage of responses and the median of response latencies, as functions of both the interval between two 2.5-msec flashes (unfilled circles) and the duration of one flash (filled circles). Points for one 2.5 -msec flash are plotted with both symbols. All flashes were equal in luminance. Experiment 3.

long latencies that occurred at long intervals; it gives the upper halves of cumulative frequency distributions. The experiments are described separately.

In Experiment 1, the $S$ always responded to each stimulus. While the percentage of responses was uniformly $100 \%$, the latency of response increased gradually to an asymptote as the interflash interval increased (Fig. 1). The asymptote, reached when intervals were longer than $50 \mathrm{msec}$, was equal to the latency for the first flash alone. Figure 4 (first panel) shows that the distribution of latencies for the first flash accounts for the distributions obtained when the two flashes were well spaced temporally.

In Experiment 2, the percentage of responses ranged between $80 \%$ and $100 \%$. Although Bloch's law was obeyed by percentages when the stimulus was brief, the stimulus energy alone did not account for the associated response latencies. In particular (Fig. 2), two-flash stimuli of the same energy yielded percentage functions that superposed but latency functions that were inversely related to the stimulus luminance. Moreover, increasing the interflash interval had no influence on the percentage when the interval was short but produced increases in the latency; it reached the value for the first flash alone when the interval was near $50 \mathrm{msec}$. At intervals longer than $50 \mathrm{msec}$, however, the percentage decreased monotonically to $85 \%$, the value for the first flash alone. Meanwhile, the latency continued to increase to a peak that was longer than the value for the first flash, after which it returned to the first-flash value. In Fig. 4, the nonmonotonicity in the latency function is expressed by the scatter of long-interval latencies to the right of first-flash latencies; the effect was more pronounced when the stimuli comprised 2.5-msec flashes (third panel) instead of 25-msec flashes (second panel). In both cases, a small proportion of long-interval latencies were considerably longer than any for the first flash.

In Experiment 3, the percentage of responses to the two-flash stimuli ranged between $20 \%$ and $80 \%$. When the stimulus was brief, the energy in the stimulus was an adequate basis for predicting percentages, in accordance with Bloch's law. But not all the corresponding latencies were in line with the law. In particular (Fig. 3), equal-energy stimuli, which comprised one 5 -msec flash and two briefly spaced 2.5-msec flashes, yielded equal percentages, but the former stimulus produced a longer

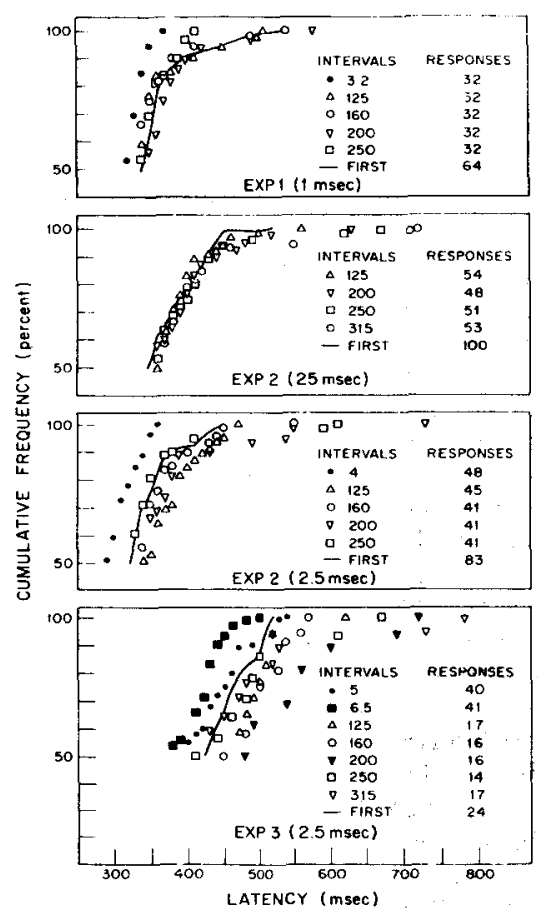

Fig. 4. The upper halves of cumulative frequency distributions of latencies in the three experiments. Each panel is for one pair of flashes whose duration is indicated in parentheses. The curve is the distribution for the first flash of the pair; the unconnected points are the distributions for the pair at the interflash intervals given in the legend. The legend also gives the number of responses in each distribution. Each distribution plots the percentage of latencies briefer than limiting values taken in 10-msec steps; however, they were smoothed by plotting one point at the highest step for which a given percentage occurred. 
latency. The percentage remained at about $70 \%$ until the interval between the two flashes was increased to about $40 \mathrm{msec}$. By this time, the latency had increased gradually to the value for the first flash alone. At longer intervals, the percentage decreased to a steady value of $30 \%$, which was above the $22 \%$ obtained with the first flash alone. Meanwhile, the latency continued to increase to a peak that was much longer than the first-flash value, to which it finally returned at the longest intervals. The nonmonotonicity in the latency function is clear in Fig. 4 (fourth panel). There one also sees that the distributions for long intervals contain a small proportion of latencies that are very much longer than any in the distribution for the first flash. For example, latencies of nearly $800 \mathrm{msec}$ occurred when the interval was $315 \mathrm{msec}$.

\section{DISCUSSION}

Viewed with reference to either the percentage or the latency of response, the interaction between the two successive flashes decreased as the interval between them increased. The percentage displayed the variations seen in earlier threshold studies, where Bloch's law held at short intervals, and the outcome was more variegated at long intervals. Meanwhile, other facets of the underlying processes were brought to the surface via the latency functions. How the two response measures covaried is relevant to a number of questions in the literature, such as those regarding the mechanisms responsible for Bloch's law. The experiments are, moreover, likely to suggest directions for future work, because of their intriguing disclosures.

\section{Short Intervals}

When intervals were shorter than $50 \mathrm{msec}$, increasing the interval had little influence on the percentage of responses, as in the experiments by Bouman and van den Brink (1952). In general, the percentage was predictable with respect to the stimulus energy alone, in accord with Bloch's law.

The accompanying latencies increased gradually to the value obtained when only the first of the flashes was presented. Latencies showed this relationship in all the experiments, pointing to an invariance. Furthermore, one- and two-flash stimuli of a constant energy yielded latencies that were inversely related to the stimulus luminance (Experiment 2), in agreement with Grossberg's (1968) findings. The latency thus varied as a function of not only the stimulus energy, but also its distribution in time; responses were emitted more rapidly the sooner the energy impinged after stimulus onset. These modified threshold experiments, plus Grossberg's, appear to be the only ones in the literature where the temporal distribution of the stimulus had observable effects while Bloch's law held and visual adaptation was fixed (Graham, 1965, p. 77; Kintz \& Boynton, 1969). The findings are apt to the interpretation of Bloch's law.

One plausible hypothesis assumes, parsimoniously, that "a finite integration period, triggered by the first quantum of light absorbed, persists for some critical duration during which all quanta are 'counted,' with a response subsequently being produced in proportion to this number [Boynton \& Siegfried, 1962, p. 721]." Accordingly, when a constant stimulus energy is presented during a period of time equal to the critical duration (i.e., the briefest flash duration for which luminance-duration reciprocity holds under given conditions), the temporal distribution of the energy should have no behavioral effect; initial integration would erase temporal information in the stimulus. Strong support has come from threshold experiments in which the S's visual adaptation was fixed; the temporal distribution had no discernible effect (Graham, 1965, p. 77). Nonetheless, the hypothesis has been questioned for a variety of reasons (Blackwell, 1963,p. 154; Boynton \& Siegfried, 1962; Kahneman \& Norman, 1964; Kintz \& Boynton, 1969). The functional dissociation of the frequency and latency of response in the present experiments is very strong evidence against the validity of the hypothesis. Instead, the processes mediating the response appear to involve an initial temporal weighting of the input, as a number of investigators have already proposed (e.g., Blackwell, 1963; Kintz \& Boynton, 1969; Raab, 1962, p. 589). Why the latency is more sensitive than the frequency to the stimulus waveform is a challenging question. Any answer would need to be consistent with the results at long interflash intervals too.

\section{Long Intervals}

At intervals longer than $50 \mathrm{msec}$, the percentage of responses decreased monotonically to a steady level, as one might expect from some studies (Bouman \& van den Brink, 1952; Davy, 1952); others showed a nonmonotonic trend (e.g., Ikeda, 1965). The asymptote was either (1) equal to (Experiment 2) or (2) higher than (Experiment 3) the percentage for the first flash alone. The first asymptote is analogous to the result at the longest interval in Davy's (1952) threshold experiment, where the energy in the two-flash stimulus was double the energy required when only one flash was presented. The second asymptote is similar to the outcome obtained by Bouman and van den Brink (1952), who accounted for it in terms of probability summation. Assuming that the flashes afforded independent opportunities to detect the stimulus, the expected relative frequency was: $1-(1-P)(1-P)$, where $P$ is the relative frequency for one flash. Accordingly, the first asymptote here (85\%) signifies no probability summation (given $85 \%$ for the first flash); however, the second asymptote (30\%) signifies about half the theoretical amount (39\%, given $22 \%$ for the first flash). It is noteworthy that these different asymptotes occurred under the same viewing conditions, and that the experiments by Bouman and van den Brink and by Davy were also performed under similar viewing conditions. Judging from the present findings, the relative effectiveness of their first flashes may have had something to do with the difference between their asymptotes. Further insight into this complex state of affairs is given by the latency data.

The corresponding latencies either (1) equaled the value for the first flash alone (Experiment 1) or (2) increased from the first-flash value to a peak and then returned to the first-flash value (Experiments 2 and 3). Thus, the two flashes sometimes produced a longer latency than the first alone, indicating that the second flash caused a delay in response emission. This prolongation appears to be another manifestation of the inhibitory interaction that was inferred by Ikeda (1965), when neither energy summation (exemplified by Bloch's law) nor probability summation (requiring independence) between the flashes was evident.

The interaction was absent in the experiments by Bouman and van den Brink. It would account for the absence of probability summation in Davy's experiment, as well as the two asymptotic percentages here. In the present work, a less effective first flash was associated with more probability summation and, presumably, less inhibition. It thus seems that there was both inhibition and probability summation at certain intervals, just as Blackwell (1963, p. 156) has hypothesized. This mixture of mechanisms, which is difficult to analyze with reference to the response percentage alone, is strongly suggested by the latency distributions in Fig. 4 (fourth panel).

Consider this hypothetical case. If the $S$ "missed" the first flash, but responded to 
the second instead, then the latency might be equal to the latency for the first flash plus the interflash interval. If, for example, the 315-msec interval is subtracted from the nearly 800 -msec latencies obtained with it, the remainder falls within the range of the distribution for the first flash. Whether the second flash was responded to independently of the first (probability summation) or displaced the first (a form of inhibition) cannot be distinguished rigorously in these data; a further juggling of percentages and latencies in order to classify the response by mechanisms seems unwarranted. Nonetheless, the intuitive analysis suggests that the two mechanisms operated in a confounded manner over trials. It encourages the belief that latencies can eventually provide a more detailed picture of the mechanisms underlying response on subsets of trials.

\section{REFERENCES}

BLACKWELL, H. R. Neural theories of simple visual discrimination. Journal of the Optical
Society of America, 1963, 53, 129-160.

BOUMAN, M. A., \& van den BRINK, G. On the integrate capacity in time and space of the human peripheral retina. Journal of the Optical Society of America, 1952, 42, $617-620$.

BOYNTON, R. M., \& SIEGFRIED, J. B. Psychophysical estimates of on-responses to brief light flashes. Journal of the Optical Society of America, 1962, 52, 720-721.

DAVY, $E$. The intensity-time relation for multiple flashes of light in the peripheral retina. Journal of the Optical Society of America, 1952, 42, 937-941.

GRAHAM, C. H. Some fundamental data. In C. H. Graham (Ed.), Vision and visual perceprion. New York: Wiley, 1965.

GROSSBERG, $M$. The latency of response in relation to Bloch's law at threshold. Perception \& Psychophysics, 1968, 4, 229-232.

IKEDA, M. Temporal summation of positive and negative flashes in the visual system. Journal of the Optical Society of America, 1965, 55, 1527-1534.

KAHNEMAN, D., \& NORMAN, J. The time-intensity relation in visual perception as a function of observer's task. Joumal of Experimental Psychology, 1964, 68, 215-220.

KINTZ, R. T., \& BOYNTON, R. M. Temporal summation during backward visual masking. Journal of the Optical Society of America $1969,59,212-216$

RAAB, D. H. Statistical facilitation of simple reaction times. Transactions of the New York Academy of Sciences, 1962, 24, 574-590.

van den BRINK, G., \& BOUMAN, M. S. Variation of integrative actions in the retinal system: An adaptational phenomenon. Journal of the Optical Society of America, 1954, 44, $616-620$.

\section{NOTES}

1. These experiments were performed in partial fulfillment of the requirements for the doctoral degree at Brooklyn College of the City University of New York. The author is indebted to Dr. E. G. Heinemann, his sponsor, and wishes to thank Drs. W. S. Battersby and W. H. Ittelson, members of the dissertation committee. This paper was prepared at Lincoln Laboratory, where the author's colleagues in the Psychology Group, Dr. W. P. Harris, Mr. O. G. Selfridge, and Dr. D. B. Yntema, were very helpful with their suggestions regarding the expository style.

2. Present address: Massachusetts Institute of Technology, Lincoln Laboratory B-267, P.O. Box 73, Lexington, Massachusetts 02173.

(Accepted for publication August 27, 1969.) 